\title{
Anthropologie de la postmondialisation
}

\section{Raymond Mayer}

\section{OpenEdition}

\section{Journals}

Édition électronique

URL : https://journals.openedition.org/pa/1868

DOI : 10.4000/pa.1868

ISSN : 2273-0362

\section{Éditeur}

Université Lumière Lyon 2

\section{Édition imprimée}

Date de publication : 1 janvier 2005

Pagination : 24-34

ISBN : 1634-7706

ISSN : 1634-7706

\section{Référence électronique}

Raymond Mayer, «Anthropologie de la postmondialisation », Parcours anthropologiques [En ligne], 5 | 2005, mis en ligne le 06 juillet 2021, consulté le 21 juillet 2021. URL : http://journals.openedition.org/ pa/1868; DOI : https://doi.org/10.4000/pa.1868 


\section{ANTHROPOLOGIE DE LA POSTMONDIALISATION}

Même s'il est dans mes intentions de créer une anthropologie du virtuel, voire une anthropologie virtuelle, cet article n'a rien d'une anthropologie-fiction. Il ne cherche pas à considérer comme révolu ce qui est précisément conçu comme non révolu et donc explicitement reconnu comme un processus en cours. Tout juste se permet-il de prendre parti sur les deux termes possibles de la "mondialisation", et cela sur un plan spécifiquement anthropologique. À savoir : l'aboutissement de la mondialisation donnera soit une uniformisation in fine des cultures, soit au contraire leur démultiplication à l'infini par le jeu des métissages culturels cumulés. De ce point de vue, il s'agit moins pour une science de s'autoriser à anticiper sur des situations inédites que de vérifier la vertu prédictive de ses conclusions théoriques courantes.

Pour ce faire, je propose de réévaluer en un premier temps l'inscription de toute anthropologie dans une conscience du "monde", en partant des inspirations fondatrices de Morgan et Murdock, et de leurs relectures par Terray, Augé et Godelier. En un second temps, je ré-examinerai la position de la génétique des populations par rapport à la question de l'unité du psychisme humain dans le but de reconfigurer les liens entre homme biologique et homme social en fonction de la pluralité des cultures. Leroi-Gourhan, CavalliSforza et Lévi-Strauss me fourniront ici des clés de lecture pertinentes. Enfin, j'explorerai les deux hypothèses postulées comme termes théoriques de la mondialisation pour montrer quelles cohérences les lient l'une à l'autre dans la construction de toute "postmondialisation " possible. Là mes interprètes de référence seront Warnier, Goody et Sahlins, l'idée générale soutenue étant que la science qui pense la mondialisation est «naturellement " capable de penser la postmondialisation.

\section{L'inscription de l'anthropologie dans la conscience du « monde »}

L'anthropologie a-t-elle précédé la mondialisation actuelle comme conscience anticipée, on pourrait presque dire : "instinct du monde " ? Si tel est le cas, l'a-t-elle fait en connaissance de cause ou à son insu? Revenons pour nous en assurer aux premiers actes anthropologiques posés par Morgan et Murdock. Quelle qu'ait été la prégnance du genre monographique dans l'essor ultérieur de la discipline, il est utile de se rappeler, en remontant le cours de la science vers ses origines les plus marquantes, que Lewis Henry Morgan publia dès 1870 à la Smithsonian Institution de Washington son livre Systems of Consanguinity and Affinity of the Human Family dont le titre stipulait d'emblée que le débat anthropologique se situait à l'échelle de la "famille humaine ". Ce qui l'autorisait à disposer d'un tel titre n'était pas d'avoir mené une enquête de terrain chez les Iroquois, mais d'avoir envoyé près de mille copies d'un questionnaire sur les terminologies de parenté et les règles de mariage à des missionnaires, des fonctionnaires et des administrateurs coloniaux répartis à travers le monde entier (Trautmann 1987 cité par Godelier 2004, 20). Dès les commencements, était tracée et imposée une perspective mondiale.

À partir de cet exemple originel, on pourrait arguer que l'anthropologie est d'une certaine manière un acte constitutif de mondialisation, et que la mondialisation est pour le moins inscrite dans tout projet anthropologique. Il ne s'agit plus ici de la mondialisation en anthropologie, mais de la mondialisation par l'anthropologie. L'acte inaugural de l'anthropologie est un acte inaugurant le monde planétaire. Ce n'est pas pour rien que l'ethnologie continue, plus d'un siècle après sa fondation, à être qualifiée en termes plus ou moins crus de "science exotique ". À condition de remarquer que l'altérité de l'anthropologie n'est pas une distanciation - concept dans lequel se complaisent pourtant les gloses habituelles - mais qu'elle est d'avance une construction de la mondialisation.

Le «commencement » de Morgan a "gravé » la mémoire anthropologique de manière fondamentale et durable. En passant de la terminologie iroquoise de la parenté à des milliers d'exemples de nomenclature, il ne passe pas simplement de l'un au multiple, mais il inaugure "dès les débuts " une tension entre le local et le mondial. Il n'additionne pas seulement des cas, mais il les classe et de ce fait il ouvre une problématique. On doit même dire qu'il ouvre plusieurs problématiques: le "monde entier» (le monde conçu comme totalité) ne sert pas simplement à confirmer la typologie iroquoise, mais à diversifier la question des nomenclatures, et au-delà de la diversification, à interroger la causalité de la diversification, et donc à poser le mode d'êtreau-monde à la fois comme un et divers. Le processus n'est pas un processus de généralisation, mais un passage à 
l'exhaustivité intelligible. Quand on dit que le terrain de l'anthropologue commence au village mais s'arrête au monde entier, on ne dit pas que l'on s'arrête faute de combattant, mais on cherche à arrêter une opinion en construisant de l'universel. L'arrêt n'est pas la complétude de l'enquête, mais la vérification par la mondialisation de la pertinence du cas particulier qui se présente au regard de l'ethnologue. La fameuse méthode inductive de l'anthropologie a comme horizon véritable une déduction qui se confond avec l'exhaustivité du « monde entier ».

Ce n'est donc pas un hasard si le Morgan auteur d'observations particulières sur les Iroquois et d'observations étendues sur les nomenclatures de parenté est le même que celui qui inaugure la mondialisation verticale dans Ancient Society. "Mondialisation verticale» est probablement l'une des expressions qui pourrait le mieux traduire la diachronie de l'évolutionnisme inventé par Morgan et la hiérarchisation intrinsèque qui l'accompagne. Les conceptions de Morgan ont été déclarées obsolètes pour Ancien Society et valables pour Systems of consanguinity par les commentateurs attitrés de l'anthropologie. Emmanuel Terray est l'un des rares commentateurs à avoir osé le contrepoint du commentaire entendu qui faisait de Morgan la "victime d'un étrange dédoublement " (1972, 15). Commentant le commentaire de LéviStrauss sur Morgan, il remarque que ce dernier applaudit le Morgan de 1870 et fustige le Morgan de 1877. Si je me permets de tarder sur l'exemple de Morgan, c'est qu'il traduit exactement, dans ses opinions, l'opinion populaire encore dominante en Occident centvingt ans après lui. L'anthropologie a beau jeu de balayer l'évolutionnisme par le diffusionnisme et le fonctionnalisme : il n'est pas un Occidental, y compris dans la sphère scientifique, qui ne soit intimement conditionné par le fait que l'évolution technologique est pensée comme un indicateur du niveau culturel, et dans une expression plus mathématique, comme "gradimètre " du niveau de conscience des cultures. La science occidentale, exactement comme "l'esprit capitaliste " chez Max Weber, est le critère ultime de toute épistémologie possible. Qu'on le veuille ou non, l'opinion commune du début $\mathrm{du} \mathrm{XXI}^{\mathrm{e}}$ siècle est à l'unisson avec l'opinion scientifique de Morgan de la fin du XIX ${ }^{\mathrm{e}}$ siècle. Interrogeons-nous tout de même quelques instants sur ce troublant isomorphisme !

Doit-on poser l'hypothèse d'une bonne "communication» de Morgan, qui aurait réussi à laisser son testament davantage auprès du grand public qu'auprès du public scientifique? Ou la "diffusion " des idées de Morgan est-elle le résultat de la lenteur d'une infusion ? Ce serait sans doute trahir l'histoire des idées que de ne pas considérer que c'est le darwinisme et non le morganisme qui s'est imposé à la conscience scientifique mondiale et donc que ce sont des doctrines d'inspiration génétique qui ont creusé le sillon des sciences $\mathrm{du} \mathrm{XX}^{\mathrm{e}}$ et du $\mathrm{XXI}^{\mathrm{e}}$ siècle et non les doctrines de source anthropologique, encore que dans ce cas précis, les deux aillent de conserve. La prévalence de la génétique dans la pensée du devenir du genre humain est la raison qui me pousse à réexaminer plus loin la corrélation jamais éteinte entre le biologique et le social.

Si nous tenons Morgan pour le créateur d'une "mondialisation verticale » dès le XIX ${ }^{\mathrm{e}}$ siècle, il nous faut aussi revenir sur ce qui par voie de contraste peut être considéré chez lui comme une "mondialisation horizontale». Regardons de plus près comment Morgan passe de l'Iroquois aux exemples du "monde entier». Il ne s'agit pas de reconstituer une anecdote, mais le processus par lequel l'anthropologie, en se construisant, construit la mondialisation. Il ne passe pas immédiatement de l'Iroquois aux questionnaires généralisés. La médiation s'est faite par la contrariété du système iroquois d'avec le système qui ne s'appelait pas encore " eskimo " mais qui est bien le nôtre, du moins le système européen dominant. Et la médiation s'est poursuivie avec la " comparaison restreinte » avec l'un ou l'autre système ni iroquois, ni eskimo : en tout cas un tiers (non exclu). Ce que nous appelons aujourd'hui couramment dans nos principes méthodologiques une pré-enquête. Ce n'est qu'après une ou deux contrariétés, au sens logique comme au sens psychologique, que Morgan mondialise! Autrement dit, le passage du local au mondial s'inscrit dans une série d'opérations intermédiaires, qui sont au minimum duelles, sinon tierces. La "globalisation », si l'on préfère l'étiquetage anglo-saxon de la "mondialisation ", n'est jamais immédiate, mais médiate. De là l'impression courante que l'infiniment grand échappe à la conscience de l'infiniment petit, généralement représenté dans un tel univers de référence par les cas individuels.

Au commentaire de Terray, le commentaire de Marc Augé ajoute la dimension temporelle, ce qui n'est pas la même chose que la diachronie. A travers son titre culte "Pour une anthropologie des mondes contemporains" (1994), la mondialisation de Marc Augé n'est plus l'exhaustivité des cultures, mais leur mise en contemporanéité. La formule-type est que les "sociétés traditionnelles" sont désormais inscrites dans la même contemporanéité que nos propres sociétés. La diachronie se résorbe dans la synchronie. Il y a eu "rattrapage". L'histoire des uns a rattrapé l'Histoire des autres. Cette mise en contemporanéité est un pur effet de «mondialisation ». L'intérêt de la formule est qu'elle implique une autocritique de l'anthropologie : l'anthropologie de Morgan avait, malgré son vaste regard encyclopédique, postulé une hiérarchie des cultures; l'anthropologie de la mondialisation mûrement réfléchie positionne des cultures "contemporaines", sans préjuger d'une quelconque hiérarchie. Le temps était donc le seul écart entre les cultures. La hiérarchie des cultures n'avait pas d'autre fondement que l'écart temporel : il suffisait d'attendre ! 
Cependant l'emploi du pluriel «mondes contemporains " atteste paradoxalement d'une négation de la mondialisation au moment même où on semblerait la poser. Car la mondialisation postule la convergence et la "globalisation ", terme invariablement donné comme son synonyme anglo-saxon et posé comme son alternyme simple. Est-ce reculer pour mieux sauter? Il serait étonnant que l'auteur de Non-lieux et de l'anthropologie de la «surmodernité » (1991) manque sa cible, au point de rater la «mondialisation » au moment même où il cherche à la définir ! Je fais le pari d'interpréter Augé dans le sens de la deuxième issue possible de la mondialisation, c'est-à-dire celle de la juxtaposition de "cultures éclatées » et de "cultures métisses " à l'infini. Les «mondes contemporains » traduiraient ainsi l'hétérogénéité attendue des cultures au terme du processus en cours.

Le postulat comparatiste de Morgan reste le postulat de l'anthropologie. C'est la raison pour laquelle je prends la peine de "rabâcher" Morgan. Le cheminement intellectuel de Morgan, que l'on ne s'y trompe pas, est peu ou prou le cheminement de chaque anthropologue, et peu ou prou le cheminement séculaire de l'anthropologie dans son ensemble. La phylogénie anthropologique suit le modèle de l'ontogénie anthropologique, et réciproquement! Les monographies ont parsemé la route de l'anthropologie tout aussi sûrement que les théories y moissonnent. On accréditera en ce sens l'idée de Clifford (1996, 33) selon laquelle la monographie ethnologique est « une fusion (...) de la théorie générale et de la description empirique ". Le nombre d'anthropologues débutant autrement que par un terrain ou poursuivant autrement que par comparaison doit correspondre à un ensemble vide.

$\mathrm{Au}$ terme du "devoir d'inventaire ", reconnaissons donc que Morgan continue à exercer sur l'anthropologie une sorte de fascination. La raison en est que les problèmes qu'il a posés ne sont toujours pas résolus. Nous avons hérité d'un constat de diversité terminologique et d'une esquisse typologique des nomenclatures de parenté, mais nous ignorons toujours la cause des transformations d'une terminologie. À commencer par celle qui concerne la transformation de notre propre système terminologique de parenté à l'époque latine. Comme le rappelle Godelier qui lui aussi relit Morgan pour analyser les Métamorphoses de la parenté $(2004,198)$, le premier système terminologique latin fut de type soudanais (c'est-à-dire pluri-linéaire), et il fut remplacé au deuxième siècle après Jésus-Christ par le type eskimo (à lignée directe unique) que nous connaissons, sans que nous arrivions à reconstituer la cause de cette substitution typologique. De plus, malgré plusieurs changements notoires de systèmes économiques passant du servage au capitalisme, le système eskimo est demeuré inchangé dans notre propre société, alors qu'il semble s'imposer progressivement au monde entier pour des raisons que l'individualisation forcée n'explique pas entièrement. Bref, la mondialisation verticale et horizontale de Morgan nous laisse avec autant de questions que de réponses.

L'entreprise anthropologique de Morgan a été confirmée par la dimension mondiale prise par les Human Relations Area Files dont Murdock a pris l'initiative soixante ans plus tard et qui restent emblématiques d'une discipline qui situe son champ de comparaison à l'échelle planétaire, quand bien même seul un nombre infinitésimal d'anthropologues embrasse ce niveau. En accolant le nom de Murdock à celui de Morgan, nous n'avançons pas seulement dans l'ordre chronologique, mais aussi dans l'ordre épistémologique. En effet, la constitution d'un fichier universel et multithématique au lieu d'être dédié aux seules terminologies de parenté est plus qu'un aveu de méthode, c'est une prise de position sur les tenants et les aboutissants de l'anthropologie comme science, capable de traiter le monde et tous les aspects du monde, à condition que ceux-ci aient été maîtrisés par leur mise en fiches! "La particularité des HRAF, écrit Marc Kurt Tabani (2001), est sans doute qu'ils ne recherchent pas l'exhaustivité des cultures mais une systématicité dans les moyens d'interrogation de leur diversité. En 1988, ils concernent 330 ethnies ou unités culturelles, et analysent environ 6100 sources, ce qui représente 740000 pages de texte dépouillé et 3800000 de fiches classées " (d'après Chevallier-Schwartz 1992, 342).

À partir des initiatives pérennes de Morgan et Murdock, l'anthropologie se projette définitivement comme science de la totalité des cultures humaines, même si le référent des anthropologues individuels débute dans les limites d'un premier terrain. Plus que d'autres disciplines, l'anthropologie traduit ses résultats dans la complémentarité totalisante des terrains investigués et dans la vérification des théories émises par la somme des terrains. Sur ce plan, elle a eu avec la géographie une dimension prométhéenne, entraînant dans leur double sillage l'ensemble des sciences exactes et une partie des sciences humaines. $\mathrm{Au}$ terme de cette première étape, considérons que l'anthropologie fait corps avec la mondialisation, fera-t-elle corps aussi avec la postmondialisation?

\section{L'unité du psychisme humain et l'anthropodiversité}

Pourquoi rapporter le débat de la mondialisation par l'anthropologie au terrain biologique? Parce qu'une fois encore, il nous ramène aux origines de l'anthropologie, cette fois-ci conçue comme science de l'évolution humaine et s'organisant avec les outils de l'anthropologie physique (aspect qui a été maintenu dans la conception américaine de l'anthropologie) avant de devenir "science sociale ". L'association délibérée dans nombre de projets actuels de l'anthropologie avec la génétique des populations est en quelque sorte 
un retour aux sources, si l'on ne veut pas perdre de vue que les dénominateurs communs tributaires de la phylogenèse constituent à la fois la base de départ et l'horizon de la recherche anthropologique.

Dans ce type d'approche, la question laissée ouverte par Leroi-Gourhan sur «la diversification des ethnies » dans Le geste et la parole de 1964 (p. 200) est décisive pour notre appréciation de l'ampleur du phénomène que nous décrivons sous le terme de «mondialisation». Si le "fractionnement en ethnies de l'espèce zoologique humaine » (ou dans la phraséologie de Leach $(1980,365)$ la "non-unité sociale» opposée à "l'unité de l'homme en tant qu'espèce ») était à la fois le moteur et le postulat de l'histoire, alors la mondialisation conçue comme uniformisation des cultures marquerait pour la première fois un reflux des cultures, c'est-à-dire une inversion inédite de tendance dans une dynamique jusque là orientée dans le sens de la différenciation. Après le flux des cultures caractéristique de la prémondialisation, il y aurait maintenant un reflux des cultures caractéristique de la mondialisation. L'anthropodiversité, à l'exemple de la biodiversité, entrerait dans une phase récessive. Une telle hypothèse serait évidemment maximaliste et pourrait nous faire concevoir la postmondialisation comme un retour au flux différenciatoire des cultures. Dans ce débat, les points de repère changent évidemment d'échelle et nous obligent à une comparaison avec des phénomènes auxquels nous sommes habituellement étrangers. Nous passons d'une échelle temporelle incluse dans la sphère anthropologique à une temporalité qui lui est extérieure, se situant à l'échelle biologique et non plus à l'échelle sociale (ou culturelle). Cette nouvelle échelle nous permet de mieux apprécier l'importance relative ou absolue de la "mondialisation " au regard non pas des ères géologiques, mais du moins de l'histoire de l'anthropisation.

À ce niveau, l'horizon technologique comme dépassement des limites biologiques est à prendre en compte, si l'on considère que la plus importante "révolution " humaine est celle du néolithique, c'est-à-dire celle qui a instauré le contrôle de la nature par l'invention de l'agriculture et par la domestication des animaux. Ceci nous ramène à un horizon de 10.000 ans, ce qui est évidemment loin des systèmes de causalité couramment étudiés dans des termes infiniment plus courts par l'anthropologie "sociale". Comme le note justement le généticien Cavalli-Sforza $(1994,287)$ «les modes de transmission culturelle sont particulièrement utiles à étudier quand on considère l'évolution culturelle sur le long terme; ils présentent moins d'intérêt pour le sociologue, qui s'attache à décrire des situations présentes ou qui analyse des changements à très court terme ». Autrement dit, sur le plan temporel, convenons que la référence à la biologie nous amène bien à un changement d'échelle temporelle.
«Depuis son apparition, il y a cent mille ans, l'homme moderne (souligné par nous) a changé ; il s'est différencié, constituant peu à peu ces groupes que l'on observe encore aujourd'hui sur la Terre, même si des milliers sont en voie d'extinction" (1994, 345). Remarquons que pour un préhistorien, "l'homme moderne" i.e. Homo sapiens, n'a jamais été celui de la période post-renaissance instituée par l'historien occidental. L'échelle temporelle est donc cruciale pour évaluer $a$ priori le niveau d'évolution que nous recherchons pour étalonner le passage de la mondialisation à la postmondialisation. Comme nous le verrons plus loin, un tel passage doit lui-même être rapporté au passage entre prémondialisation et mondialisation, si nous voulons respecter l'échelle des temps et mesurer le deuxième «passage" à l'aune du précédent. Mais " changer le hardware, c'est-à-dire notre bagage génétique héréditaire, serait très difficile; il est beaucoup plus facile d'améliorer le software, c'est-à-dire la culture » (1994, 345). Dans cette perspective, on voit que la génétique pousse encore plus loin l'échelle temporelle que ne le fait l'anthropologie : la seconde serait à la première ce que la variation est à la permanence.

Que nous apporte la génétique dans le débat des cultures? Elle nous reporte au débat non résolu de l'inné et de l'acquis, qui est évidemment un des modèles possibles de la compréhension de la mondialisation. Que serait la mondialisation sinon le passage de l'acquis à une sorte d'inné culturel permanent par gommage des différences? Sans avoir besoin d'entrer dans le débat génétique, je propose de prendre en considération pour comprendre la mondialisation certains aspects énoncés par Leroi-Gourhan qui depuis sa chaire de paléontologie du Collège de France pouvait pérorer sans crainte d'être contredit sur l'évolution biotechnique de l'humanité : «On peut prouver que l'équilibre matériel, technique et économique influence directement les formes sociales et par conséquent la manière de penser, alors qu'il n'est pas possible d'ériger en loi que la pensée philosophique ou religieuse coïncide avec l'évolution matérielle des sociétés. " (1964, 208). Nul ne songeait à l'accuser de biologisme. "La société façonne son comportement avec les instruments que lui offre le monde matériel; les assurances sociales ne sont pas plus imaginables chez les chasseurs de mamouths que la famille patriarcale n'est pensable dans une cité industrielle» ${ }^{2}(1964,209)$. Le primat du techno-économique sur le social et la nonréversibilité des termes de cette équation nous engagent directement dans le débat de la postmondialisation, et nous renvoient aux termes d'un débat engagé en 1952 par Claude Lévi-Strauss sur les rapports entre « race et histoire». 
«Si à certaines époques et en certains lieux, des cultures "bougent" tandis que d'autres "ne bougent pas ", ce n'est pas en raison d'une supériorité des premières, mais du fait que des circonstances historiques ou géographiques ont induit une collaboration entre des cultures non pas inégales (rien ne permet de les décréter telles), mais différentes. Elles se mettent en mouvement en s'empruntant ou en cherchant à s'opposer les unes aux autres. Elles se fécondent ou se stimulent mutuellement. Tandis qu'en d'autres périodes ou en d'autres lieux, des cultures qui restent isolées comme des mondes clos connaissent une vie stationnaire» $(1984,205)$. Si l'interculturalité est pensée sur ce modèle lévistraussien, on saisit que la mondialisation n'invente aucune règle anthropologique nouvelle, sinon la "collaboration» accrue entre cultures différentes. On pourrait même penser qu'elle réduit simplement les derniers "mondes clos". De ce point de vue, notre détour par l'anthropologie phylogénétique ajoute pour ainsi dire à l'échelle des temps une échelle des espaces.

Dans un texte de 1971 consacré à " race et culture ", le même auteur revient sur sa conception de la diversité culturelle : "chaque culture se développe grâce à ses échanges avec d'autres cultures. Mais il faut que chacune y mette une certaine résistance, sinon, très vite, elle n'aurait plus rien qui lui appartienne en propre à échanger. L'absence et l'excès de communication ont l'un et l'autre leur danger ". Ainsi sont fixées les règles d'une sorte de struggle for life des cultures : « il faut consentir à en payer le prix : à savoir, que des cultures attachées chacune à un style de vie, à un système de valeurs, veillent sur leurs particularismes ; et que cette disposition est saine, nullement - comme on voudrait nous le faire croire - pathologique » $(1984,207)$. Voici la métaphore biologique totalement transparente, et en même temps défini un danger d'excès, celui-là même que Marc Augé identifie à la "surmodernité »! Nous voilà replacés au cœur de nos définitions de la mondialisation, et par voie de conséquence, de la postmondialisation.

Au darwinisme social de Morgan dont je suis parti dans la première étape de ma réflexion, il convient d'ajouter le postdarwinisme biologique de cette deuxième étape pour définir les matrices théoriques susceptibles d'engendrer la postmondialisation à partir de la mondialisation. Que l'avenir soit dans le totalitarisme biologique d'Adlous Huxley ou dans le totalitarisme sociologique de George Orwell ne concerne d'ailleurs que le court terme. La grande leçon du détour par l'anthropologie biologique est de nous permettre de mieux fixer non seulement les repères temporels et spatiaux de la mondialisation, mais d'en cerner l'envergure par la référence à ces deux systèmes de repères. Il me semble possible maintenant de mieux poser les termes de la construction théorique de la postmondialisation.

\section{La construction de la postmon- dialisation}

À la lumière des observations précédentes, à la fois sur le plan culturel et sur le plan biologique, on comprend que l'anthropologie soit particulièrement bien placée pour traiter de la postmondialisation. Ce qui l'habilite, à mon sens, à traiter de la postmondialisation, est sa position préliminaire dans la conception de la mondialisation. Il est clair que la compétence acquise par rapport à la mondialisation est la même qui servira à penser la postmondialisation. Mais il est tout aussi clair que plusieurs constructions de la postmondialisation sont possibles, et qu'il n'est pas utile pour cela de passer par la fiction. Pour éviter et même prévenir ce jeu de «divination » risqué et stérile tout à la fois, on doit postuler plusieurs axes de recherche qui fourniront autant de lignes directrices entrant dans la définition de la postmondialisation.

J'en retiendrai ici trois, sans prétendre à une quelconque exhaustivité, mais en me servant de ces trois directions ouvertes pour précisément affirmer la diversité des solutions possibles. On peut partir ainsi d'un axe de recherche génétique et paléontologique (comme je l'ai esquissé plus haut), on peut partir d'un axe régional (et je le tenterai ici à partir d'un axe régional africain, là encore moins pour territorialiser l'entreprise que pour montrer l'emprise du local sur le général), on peut partir enfin des enjeux scientifiques globaux pour évaluer la "course» du monde et en particulier le « commerce » des idées.

3.1 S'agissant de l'axe génétique et paléontologique, il est évident que pour toute perspective sur le long terme, on peut affirmer sans crainte de se tromper que les projections vers le futur sont exactement proportionnelles aux reconstructions du passé. Plus on dispose de données sur le long terme ancien, plus on est en mesure de disposer d'outils de mesure du futur de longue portée. On doit même dire que la connaissance du passé lointain est la condition sine qua non de la connaissance du futur lointain. Le scientifique se trouve à chaque fois à équidistance des pôles à (re)construire. C'est la raison pour laquelle toute projection qui ne se construit pas sur une grande profondeur historique ne peut apparaître que comme un charlatanisme ou une imposture. Dans le cas spécifique de l'anthropologie, on comprend qu'elle est aux avant-postes, non pas par sa capacité à prédire l'avenir, mais par sa maîtrise des grandes profondeurs historiques. On voit également ce qu'il lui reste à faire pour mieux savoir ce qui se construit. Elle peut soit rester accolée à l'éminemment présent, comme les perspectives d'inspiration plutôt sociologique ; elle peut au contraire se déployer dans le sens de reconstructions historiques d'autant plus fécondes qu'elles se risqueront à frayer dans l'antique plutôt que de s'arrêter à l'histoire "moderne ". Il me reste à reprendre la question de l'unicité de l'espèce et de la pluralité des cultures telle qu'elle a été traitée 
par la tradition anthropologique pour faire entrevoir qu'elle s'applique sans transition à la postmondialisation, après avoir fait les beaux jours de la prémondialisation et de la mondialisation.

Nous avons vu pour cela que la mondialisation est inscrite au cœur de tout projet anthropologique, tant sur le plan de la méthode que de la théorie. Contrairement aux assertions courantes sur l'effet de mondialisation, la globalisation de l'économie ne signifie aucunement sur un plan scientifique la globalisation de la culture. Jean-Pierre Warnier (1999) l'a fort bien démontré dans sa mondialisation de la culture (et rappelé aussi dans sa contribution au présent numéro) où son analyse aboutissait en fait à qualifier une non-mondialisation de la culture. Je voudrais pour ma part prendre au sérieux la thèse inverse et donner du crédit à la thèse commune de la mondialisation par l'uniformisation de la culture. Nous verrons qu'en réalité deux mouvements sont à l'œuvre simultanément qui se superposent et se croisent, la contradiction ne s'avérant en définitive qu'apparente.

Il est indéniable que l'on assiste à une "mort annoncée " des langues, à une réduction drastique du nombre des langues parlées dans le monde, les quadragénaires actuels qui les parlent encore étant condamnés à disparaître à l'horizon d'un demi-siècle en emportant dans la tombe leur patrimoine immatériel irremplacable. Selon Colette Grinevald (2005), responsable d'un programme international de "langues en danger» on passera de 4000 langues à 1000 langues à l'échéance d'un siècle. Évidemment, on ne passe pas d'une langue à une absence de langues, mais on passe d'une langue ethnique à une langue véhiculaire, pour ne pas dire nationale ou internationale. Et il est peu vraisemblable que l'humanité crée dans l'intervalle une seule langue supplémentaire, comme les Afrikaaners avaient créé "leur" langue européenne en Afrique du Sud au XVII ${ }^{\mathrm{e}}$ siècle qui est restée inconnue des autres Européens. Même si la réduction linguistique est moins justiciable d'une théorie du « regroupement culturel " que du " changement culturel ", on se trouve indubitablement devant un scénario de reflux des langues, et donc de reflux culturel. Certes la mort des civilisations n'est pas une nouveauté. La civilisation égyptienne a disparu en emportant avec elle jusqu'aux clés de son écriture, qui a heureusement trouvé son Champollion. La langue latine de vivante est devenue "morte", même si elle survit partiellement dans les langues-filles romanes. Il y a eu une phase de différenciation des langues; nous serions pour la première fois dans l'histoire de l'humanité dans une phase d'homogénéisation des langues. Bref, sur le plan linguistique, nous sommes dans une phase de réduction des cultures, par choix d'assimilation à des groupes linguistiques numériquement plus importants et par choix d'extinction des langues maternelles antérieures.
De même, dans le domaine religieux, il y a incontestablement un regroupement démographique autour de "grandes religions ", même si le mouvement ne date pas d'hier. Ou alors conviendrait-il de parler de "mondialisation " déjà ancienne pour toutes les religions universalistes qui étendent intrinsèquement leur prosélytisme à toute l'espèce Homo sapiens sapiens. De ce point de vue, convenons avec Godelier qui s'en fait l'écho pour les Baruya de Nouvelle-Guinée $(1984,2004)$, que la christianisation des archipels océaniens (comprenons par là de toutes les sociétés traditionnelles, quels que soient les continents de rattachement) est, au regard de l'histoire, un événement de portée beaucoup plus importante que celle qui se reflète habituellement dans les écrits des anthropologues. Les cultes ethniques, sans disparaître totalement, sont dans une situation récessive, même si de nouveaux prophètes suscitent de nouvelles religions. Sur ce point, on comprend que le mécanisme de fondation d'une religion est très différent de celui de la création d'une langue. Autant les langues peuvent disparaître, autant les religions peuvent naître et renaître et se multiplier à l'infini. Aussi convient-il de faire la différence domaine par domaine, au lieu de vouloir comprendre l'évolution culturelle d'un seul bloc.

Procédons par positionnement extrême pour bien réaliser ce que la mondialisation ne sera pas: la mondialisation extrême serait la réduction à une seule langue, une seule religion (ou absence de religion), un seul État, une seule économie centralisée, bref une seule culture en tous points. On voit d'emblée qu'il n'y a aucune chance que ce scénario se réalise un jour, même pas sur l'un des secteurs ainsi sommairement inventoriés. Ce qui est certain, c'est que nous nous acheminons vers une «désethnicisation" de la culture. Les ethnies disparaissent au profit d'ensembles nationaux de dimensions géographique et démographique plus importantes, impliquant un imaginaire remodelé à l'image des nationalismes. Je vois en une ou deux générations fondre toute référence aux clans et aux lignages au Gabon. Notre $\mathrm{XXI}^{\mathrm{e}}$ siècle est indubitablement le siècle de la " mort des lignages " en même temps que celui de la mort des langues. Les étudiants de l'an 2000 ne connaissent plus le nom de leur patri- ou matri-lignage d'appartenance (les deux situations sont attestées au Gabon), alors que leurs aînés de 1975 les connaissaient sans l'ombre d'une hésitation. À moins que les clans ne subsistent dans l'imaginaire et dans quelques signes qui en permettent la pérennisation comme en Écosse (où les anthropologues ont d'abord découvert l'équivalent des systèmes lignagers africains), certains systèmes de parenté vont totalement disparaître au profit de formes proches de l'individu-famille, donc encore plus réduites que la famille nucléaire qui semblait être à l'orée du milieu du $\mathrm{XX}^{\mathrm{e}}$ siècle le noyau familial minimal en deçà duquel ne subsistait que l'impensable (et par conséquent l'impensé). 
Globalement, le système parental semble suivre la même évolution que le système linguistique, c'est-à-dire subir un appauvrissement numérique de ses formes.

Face au mouvement de reflux culturel existe pourtant un mouvement équivalent de nouveau flux culturel. Ce nouveau flux n'est à l'évidence plus d'origine ethnique, mais observons qu'à l'image des régions et provinces historiques européennes, des réclamations identitaires non seulement réapparaissent, mais se renforcent. À mon sens, les diasporas offrent la concrétisation parfaite de scénarios qui n'ont rien de futuriste. La fête de la St-Patrick pour les Irlandais des USA, la cuisine hispanique de la côte californienne, les communautés grecques d'Australie, les associations de Corses expatriés nous donnent une image quasi expérimentale des regroupements identitaires qui ne représentent que la face transformée de regroupements ethniques anciens. De même, les régions et "provinces historiques" européennes représentent environ deux mille ans de transformations post-ethniques, sans que les racines ethniques n'en aient été complètement extirpées. Ce type de regroupement du genre "cercle des poètes disparus" se retrouve pourtant dans une position minoritaire par rapport à une multitude de choix et de nouveaux regroupements possibles. Car les regroupements identitaires professionnels, religieux, associatifs de toute nature seront sensiblement plus nombreux que les regroupements à fondements «ethniques" anciens.

Dans tous ces cas de figure, l'individualisation inscrite dans la mondialisation ne signifie pas l'isolement des individus et ne signifie pas davantage qu'entre l'individu et l'universel n'existe plus aucune médiation. Au contraire les systèmes fédérateurs intermédiaires sont les véritables médiations des choix individuels. Chaque individu se trouve, au départ de sa vie scolaire ou active, devant une multiplicité de choix mais dans lesquels il s'investit effectivement. Autrement dit, il se trouve investi dans des cultures professionnelles, dans des cultures technologiques, dans des cultures religieuses auxquelles il n'est pas lié par la naissance, mais auxquelles il adhère pendant la durée de son existence ou pendant une partie de celleci. La nouveauté consistant à se départir d'une appartenance "sociologique » unique et à se trouver investi dans une pluralité de regroupements identitaires, là où le cadre lignager imposait un moule unique. On peut même aller plus loin en supposant que des traditions naissant dans les domaines professionnels, géographiques (cultures de terroir), religieux ou autres, on continuera à pouvoir parler de "cultures traditionnelles", mais dans un sens nouveau: il s'agira de traditions créées dans le temps en fonction des intérêts les plus diversifiés, allant d'une "chaîne des rôtisseurs " par exemple à un
Rotary-Club ou à d'autres "sociétés" à traditions. Des traditions nouvelles pourront être dénommées "cultures à traditions " comme dans les messianismes et autres mouvements fédérant des individus ou leurs groupements.

Constatant d'une part des mouvements sectoriels d'appauvrissement des cultures et d'autre part des mouvements de création de nouvelles cultures, il n'y a donc aucun paradoxe à imaginer deux mouvements simultanés, l'un d'uniformisation et l'autre de non-uniformisation des cultures. À l'uniformisation, il ne convient pas d'opposer la non-uniformisation, mais de penser la théorie de la co-existence de deux mouvements explicitement contraires. C'est pour cela que les deux thèses de la "mondialisation » sont défendables, à condition qu'on en saisisse la logique unique. On finira par admettre que c'est le face à face inédit entre l'individu (pour la première fois juridiquement élevé au niveau d'un lignage, voire d'un peuple tout entier ou d'un État, opposable par exemple à la raison d'État) et l'universel qui constitue la nouveauté radicale de la "mondialisation". Un individu n'a plus de comptes à rendre à son lignage, ni à sa famille nucléaire ou monoparentale. Il a une infinité de choix possibles entre tous les possibles qui lui sont offerts par un universel qui est à la portée du plus grand nombre, même des immigrés qui se ruent par vagues contre les barrières juridiques illusoires érigées par des nations inégales. Mais la logique d'individualisation des choix se double intrinsèquement de la création permanente de nouveaux regroupements identitaires.

De ce point de vue, la guerre entre l'homogénéité et l'hétérogénéité n'aura sans doute pas lieu. Non pas que les deux concepts ne reflètent pas deux aspects de l'avenir de la mondialisation, mais parce que la postmondialisation ne se définira pas par rapport à la seule dimension culturelle, mais par rapport à plusieurs dimensions possibles, dont présentement la dimension économique. De même que la structure impose sa loi à l'histoire chez Sahlins (1989), de même l'économie aura fini d'imposer sa loi à la culture dans la postmondialisation. La culture seule n'est pas juge de la culture postmondialisée. Il faut plus que la culture pour faire évoluer la culture. L'avenir de la mondialisation n'est donc pas le choc huntingtonien des cultures, mais le choc de l'économie et de la culture. C'est bien la raison pour laquelle on oppose les "culturalistes" aux "économistes ". Une postmondialisation se construit potentiellement, même sur le plan de ses prémisses anthropologiques, autrement que sur le seul terrain anthropologique. Ce n'est pas dire que l'anthropologie sera condamnée à courir derrière le train, mais qu'il lui sera imposé de prendre en compte des dimensions qui ne sont pas de son ressort direct, ni de son héritage indirect. 
3.2 Si l'on tente d'approcher la postmondialisation par l'anthropologie générale et régionale, on doit dire que, contrairement aux apparences, la mondialisation n'apparaît pas comme un changement d'échelle. Elle n'est que la progression logique de tous les mécanismes économiques mis en œuvre depuis le $\mathrm{XIX}^{\mathrm{e}}$ siècle. L'acception africaine de la mondialisation est à cet égard révélatrice. $\mathrm{La}$ mondialisation qui a suscité les cris d'orfraie en Europe quelques années avant l'an 2000 a laissé en Afrique une impression de "déjà vu ", et elle a créé l'expression de "deuxième mondialisation» pour bien montrer que la «première mondialisation " avait déjà un siècle d'existence. 1885, l'année de la clôture de la conférence de Berlin bismarkienne avait abouti aux règles de partage du monde entre les puissances colonisatrices, et l'Afrique avait fait, entre autres, les frais de ce partage. Si l'on suit la définition qui était ainsi engagée, la «mondialisation" n'est rien d'autre que la poursuite d'un processus de « colonisation ».

Dans le cadre de la "deuxième mondialisation ", à en juger par la conjuration massive d'un spectre qui leur semble défavorable, ce sont probablement les Européens qui se sentent poussés dans le camp des colonisés au lieu d'avoir perduré comme les maîtres de la colonisation. Pour le mondialisateur, l'avenir ne peut être que positif et aller dans le sens de l'histoire, pour les mondialisés, l'avenir ne peut apparaître que menaçant. On voit donc que la mondialisation, au lieu de ne susciter qu'un seul vécu, en suscite au moins deux. Plus fondamentalement, "l'expérience africaine " de la mondialisation permet de saisir que la mondialisation, contrairement aux apparences, n'appartient pas au futur, mais au passé. Elle appartient à l'époque précédente, pas à l'époque à venir. C'est donc la postmondialisation qui est à penser à la lumière de la mondialisation, et non la mondialisation à partir de prémisses oubliées. Voilà, s'il y en a une, la grande leçon de l'Afrique.

Autant la colonisation, du point de vue du colonisateur, ne pouvait produire que des effets bénéfiques pour tous, autant la mondialisation, du point de vue du mondialisateur, ne peut produire que des effets salutaires. Mais pour le colonisé de la première ou de la deuxième mondialisation, ce qui est pertinent dans la mondialisation ce n'est pas une projection dans l'avenir, mais au contraire le rattachement à une dynamique qui a débuté au $\mathrm{XIX}^{\mathrm{e}}$ siècle. La mondialisation nous projette en plein $\mathrm{XIX}^{\mathrm{e}}$ siècle, et non dans le futurisme du XXI ${ }^{\mathrm{e}}$ siècle. L'anthropologie qui s'est historiquement développée à la faveur de la colonisation est placée aux premières loges pour savoir de quoi il en retourne dans la mondialisation. Par voie de conséquence, la mondialisation doit être vue comme la mainmise de l'économie sur la production sociale humaine. Si Godelier dit que l'homme est le seul être à ne pas simplement être dans une société, mais à produire de la société (1984), alors on voit que la production sociale de la mondialisation ressortit à l'économie, en remplacement, entre autres, de la religion qui avait, plus qu'on ne le croit, façonné jusque là la société occidentale elle-même (Goody 1986).

Le point saillant de la mondialisation n'est donc pas l'extension des procédures, mais le fait que les procédures soient régentées par l'économique. Dans ce contexte, il est facile de voir que la postmondialisation pourrait ne pas être la fin d'un phénomène d'extension, mais la fin du primat de l'économie sur tous les autres secteurs de l'activité humaine. Les articulations entre histoire et structure, et entre culture et événement, telles que fondées par Marshall Sahlins (1989), se révèlent du plus haut intérêt, dans la mesure où elles signifieraient dans notre cas la possibilité de la fin du primat de l'économique (la structure) sur les autres secteurs de l'activité humaine (l'histoire).

La postmondialisation pourrait également être envisagée sous l'angle d'un pôle régional "tournant». Ce serait une vision minimaliste. La prise en compte de la dimension "régionale " de la mondialisation nous ferait admettre que dans une région donnée, il y aurait la possibilité de créer des "noyaux" fédérateurs, susceptibles d'une certaine manière d'échapper à la mondialisation. Partant d'une comparaison revisitée entre Orient et Occident, Jack Goody (1996 tr. fr. 1999) postule que "l'on ne peut plus considérer les grandes réalisations de l'Occident comme liées à des caractéristiques de très longue durée, voire perpétuelles, des cultures occidentales, mais comme le fruit de l'un des mouvements pendulaires qui affectent ces sociétés depuis des millénaires. L'esquisse théorique la plus élémentaire doit commencer par admettre cette alternance. " Dans cette configuration, la postmondialisation, à moins qu'il ne s'agisse de la mondialisation ellemême, pourrait consister en un "dépassement » de l'Occident par l'Orient, qui serait alors à la fois une rupture et une continuation de la mondialisation actuelle.

3.3. Que la postmondialisation doive être théoriquement une rupture par rapport à la mondialisation n'est qu'une pétition de principe. Encore convient-il d'identifier correctement de quelle rupture il pourrait s'agir. Cette rupture est à mon sens déjà inscrite dans le jeu des forces en présence et ne ressortit pas à une sorte de deus ex machina. La postmondialisation ne viendra pas par effet de mode, mais s'inscrit dans la durée aussi sûrement que l'influence des religions s'est inscrite dans la durée. Si nous prenons l'exemple de l'Islam, il est notoire qu'il n'est nullement un frein à la mondialisation actuelle. Son influence est prégnante sur le politique, mais pas sur l'économie mondiale, preuve que le primat de l'économique s'est imposé, dans le cadre de la 
présente mondialisation que nous vivons, à toute autre sphère d'influence possible.

Dans un ouvrage récent, Jean Guiart (2002, 118) fait valoir les conclusions d'une étude de Dotts-Higginson (1996) sur les paradoxes d'une société que nous reconnaissons comme éminemment mondialisatrice, la société japonaise : "le seul moyen conceptuel qui se présente à nous pour analyser la situation (japonaise) est de manier la notion occidentale de corruption des élites, ce qui n'est guère satisfaisant s'appliquant à une des économies les plus développées de la planète, mais qui s'entête à ne pas fonctionner comme le voudrait l'Occident (en ce cas représenté par le Fonds Monétaire International et plus récemment par l'Organisation Mondiale du Commerce). La société japonaise au sens large s'inspire du même principe que les sociétés océaniennes. L'institution fondamentale n'y est pas l'une ou l'autre des hiérarchies décrites par les auteurs spécialisés, mais la compétition constante entre elles. Au terme du processus, il apparaît que dans les sociétés de compétition ainsi conçues, la règle qui s'impose à tous est de ne jamais détruire l'adversaire, ce qui oblige évidemment à revoir tous les schémas conceptuels mis en œuvre pour les comprendre ». Sur cet exemple précis, on voit ce que les schémas les plus "nouveaux" doivent aux matrices culturelles les plus "anciennes". Dans ce jeu des influences respectives et des structures évolutives, il y aurait encore de nombreux enjeux scientifiques à vérifier. Dans le développement de cette expertise, l'anthropologie a certainement sa carte à jouer, y compris sur le terrain économique. Les logiques à l'œuvre dans les sociétés non mondialisées subsistent encore dans les sociétés mondialisées, ce qui confirmerait qu'il n'y a finalement pas solution de continuité entre prémondialisation, mondialisation et postmondialisation

\section{Conclusions}

À l'intérieur du champ anthropologique, principalement concerné par les présentes réflexions, il me semble utile de revenir aux deux études classiques de Claude Lévi-Strauss sur l'évolution culturelle (1952 ré-analysé dans 1984 et 1971 repris dans 1983) qui sous des titres proches abordent directement la question qui nous intéresse. "Une société ne peut se maintenir si elle n'est pas attachée inconditionnellement à des valeurs, lesquelles, pour être inconditionnelles, doivent avoir un aspect sensible qui les protège du travail de sape de la raison " $(1984,121)$. Les exemples invoqués ne proviennent pas d'une région d'Amazonie ou de Mélanésie, mais des règles protocolaires de l'Académie française et des rituels universitaires oxfordiens ! Sous-jacente à la démonstration, l'affirmation d'une logique permanente non seulement dans la rationalité, mais aussi dans le sensible, qui se perpétue malgré les déconstructions et les reconstructions successives.
La construction de la postmondialisation est vouée à obéir aux mêmes règles que celle de la mondialisation. On peut procéder par contrastes simples comme nous l'avons tenté, comme régionalisation versus mondialisation, local versus mondial, désenchantement versus réenchantement. Cette dernière opposition est d'ores et déjà construite par Jean Stone (2005) dans un ouvrage collectif consacré à la science et la quête de sens. Il y rappelle que le théorème de Kurt Gudell nous achemine à la certitude, comme dans la théorie quantique sur la position et le mouvement de l'électron et sa reprise anthropologique dans les théorisations de Georges Devereux (1985), qu'il est impossible d'être cohérent sans être incomplet, ou d'être complet sans être incohérent. Entre le principe de la complétude et celui de la cohérence, on est obligé de renoncer à la totalité des possibles en même temps. Postulant un temps et un espace qui dépassent le temps et l'espace que nous connaissons, la science ouvre un futur qui échappe à l'emprise de la science et rouvre un horizon du savoir qui réintroduit même des possibles non scientifiques du savoir. Appliqué à la postmondialisation, le théorème permet de trancher d'avance sur les aboutissants possibles et impossibles, en acceptant de limiter son ambition à quelques issues possibles sans pour autant prétendre à la complétude.

À titre de concrétisation des risques de la virtualité, on pourrait citer l'exemple littéraire posthume de Jules Verne dans son Paris au $X X^{e}$ siècle, dont on se souvient que le manuscrit oublié de 1867 ne fut édité qu'en 1994. Que n'a-t-on été surpris d'y trouver l'auteur des technologies anticipatrices du $\mathrm{XX}^{\mathrm{e}}$ siècle situer le Paris de 1967 au stade d'un "Cinquième Empire ", donc incapable d'y entrevoir une évolution politique autrement que sur le modèle interne de celle qui se déroulait sous ses yeux ! La logique de la mondialisation est fille de la prémondialisation, de même que la mondialisation sera la mère «naturelle " de la postmondialisation.

$\mathrm{Au}$ terme de ce parcours, nous pouvons admettre comme acquis que la "mondialisation» se situe dans le champ de la «prémondialisation" et comme telle se rattache non pas à la longue durée, mais à la durée historique, à l'intérieur d'un cycle d'un partage des compétences entre l'économique et le culturel (pour ne pas dire le social). Pour en revenir à la perspective tracée par Morgan dans Ancien Society, si on devait se demander si la postmondialisation ouvre un "nouvel âge " de l'humanité, on répondrait à coup sûr par la négative! La fin de la mondialisation, s'il y a en a une de tangible, ne sera pas la fin d'une extension des procédures de globalisation actuelle, mais la fin du règne du pan-économisme. On comprend tout à la fois que la mondialisation n'est pas la fin de l'histoire et que l'enjeu de la postmondialisation soit éminemment anthropologique. 
Par ailleurs, si l'on tient la logique de l'hétérogénéité culturelle jusqu'à son terme, on devra admettre que la postmondialisation sur le plan anthropologique se confond avec la mondialisation, dans la mesure où le processus commencé en Europe depuis deux mille ans se poursuit en réalité dans les autres cultures, sans que les regroupements identitaires soient susceptibles de fusionner dans le magma global d'une culture unique. Plus profondément, en raison $d u$ développement simultané de deux mouvements contraires que nous avons formellement identifiés, c'est à la fois une théorie de l'hétérogénéité et de l'homogénéisation culturelles qui lui sera différentiellement applicable.

\section{Raymond MAYER}

\section{RÉFÉRENCES BIBLIOGRAPHIQUES}

- $\quad$ Auge Marc, 1991, Non-lieux. Anthropologie de la surmodernité, Paris, Seuil.

- Auge Marc, 1994, Anthropologie des mondes contemporains, Paris, Flammarion.

- CAVAlli-Sforza Luca, 1992, Qui sommes-nous ? Une histoire de la diversité humaine, Paris, Odile Jacob.

- CllfFord James, 1996, Malaise dans la culture. L'ethnographie, la littérature et l'art au XX siècle, Paris, Ensb-a.

- Chevallier-Schwartz Monique, 1992, Human Relation Area Files dans Bonte et Izard (dir.) Dictionnaire de l'ethnologie et de l'anthropologie, Paris, PUF, pp. 342-343.

- Devereux Georges, 1985, Ethnopsychanalyse complémentariste, Paris, Flammarion.

- $\quad$ Dobbs-Higginson M. S., 1996, Asia Pacific : Its role in the world disorder, Sydney, Reed.

- GodeLIER Maurice, 1984, L’idéel et le matériel, Paris, Fayard, 349 p.

- $\quad$ Godelier Maurice, 2004, Métamorphoses de la parenté, Paris, Fayard, 665 p.

- Goody Jack, 1986, L'évolution de la famille et du mariage en Europe, Paris, Armand Colin.

- Goody Jack, 1999, L'Orient en Occident, Paris, Seuil, (The East in the West, Cambridge, 1996), $397 \mathrm{p}$

- $\quad$ Guiart Jean, 1976, Objets et mondes, Paris, Revue du Musée de l’Homme.

- Guiart Jean, 2002, Découverte de l'Océanie. Connaissance des hommes, Nouméa, Le Rocherà-la-Voile, 362 p.

- $\quad$ HocaRT Arthur M., 1935, Les progrès de l’homme, Paris, Payot.

- HuXley Adlous, 1928, Dans le meilleur des mondes, Paris. 
- $\quad$ LEACH, Edmund, 1980, L'unité de l'homme et autres essais, Paris, Gallimard.

- Leroi-Gourhan Georges, 1964, Le geste et la parole. Technique et langage, Paris, Albin Michel, $323 \mathrm{p}$.

- $\quad$ LÉvi-STRAuss Claude, 1983, Le regard éloigné, Paris, Plon.

- LÉvi-STRauss Claude, 1984, De près et de loin, Paris, Odile Jacob.

- Morgan Lewis Henry, 1871, Systems of Consanguinity and Affinity of the Human Family, Washington, Smithsonian Institute.

- Morgan Lewis Henry, 1877, Ancient society (tr. fr. La société archaïque, Gallimard).

- $\quad$ MuRDocK George Peter, 1935, Studies in the science of society, Yale University.

- $\quad$ ORwell George, 1960, 1984, Paris, Livre de Poche.

- SAHLIns Marshall, 1989, Des îles dans l'histoire, Paris, Gallimard/Le Seuil.

- $\quad$ Stone Jean (éd.), 2005, Science et quête de sens, Paris, Presses de la Renaissance.

- Tabani Marc Kurt, 2001, «Quand l'ethnologue quitte le terrain : anthropologie et bibliothèques » dans Ethnologies comparées, ${ }^{\circ} 2$.

- Terray Emmanuel, 1972, Le marxisme devant les sociétés «primitives ». Deux études, Paris, Maspéro, $175 \mathrm{p}$.

- Trautmann T., 1987, Lewis Henry Morgan and the Invention of Kinship, Berkeley, University of California Press.

- Verne Jules, 1994, Paris au XX $X^{e}$ siècle, Paris, les éditions du Cherche Midi (manuscrit de 1867).

- WARNIER Jean-Pierre, 1999, La mondialisation de la culture, Paris, Nathan (coll. "Découvertes »). 